\title{
The relationship of analogical distance to analogical function and preinventive structure: The case of engineering design
}

\author{
Bo T. Christensen \\ Copenhagen Business School, Copenhagen, Denmark \\ AND \\ Christian D. SChUnN \\ University of Pittsburgh, Pittsburgh, Pennsylvania
}

\begin{abstract}
Analogy was studied in real-world engineering design, using the in vivo method. Analogizing was found to occur frequently, entailing a roughly equal amount of within- and between-domain analogies. In partial support for theories of unconscious plagiarism (Brown \& Murphy, 1989; Marsh, Landau, \& Hicks, 1996) and Ward's (1994) path-of-least-resistance model, it was found that the reference to exemplars (in the form of prototypes) significantly reduced the number of between-domain analogies between source and target, as compared with using sketches or no external representational systems. Analogy served three functions in relation to novel design concepts: identifying problems, solving problems, and explaining concepts. Problem identifying analogies were mainly within domain, explanatory analogies were mainly between domain, and problem-solving analogies were a mixture of within- and between-domain analogies.
\end{abstract}

Analogy involves accessing and transferring elements from familiar categories in order to use it in the construction of a novel idea-for example, in an attempt to solve a problem or explain a concept (Gentner, 1998). Analogical reasoning is assumed to be a general human capacity (Holyoak \& Thagard, 1995) involved in most domains, although, perhaps most notably, in creative problem-solving domains, such as science, design, and art. Engineering design-perhaps, especially, the early conceptual stage of the design process-is one such creative domain. Design theorists (e.g., Casakin \& Goldschmidt, 1999; Goldschmidt, 2001; Roozenburg \& Eekels, 1996) have argued for the importance of analogy in design, and several design tools or techniques make extensive use of analogy, including Synectics (e.g., Gordon, 1961) and TRIZ (e.g., Terninko, Zusman, \& Zlotin, 1998). Furthermore, anecdotes concerning inventors and engineers who have made breakthrough discoveries or inventions following distant analogical transfer abound in the creativity literature. One of the most famous is George de Mestral, who developed Velcro after examining the seeds of the burdock root that had attached themselves to his dog. Regardless of whether these anecdotes are true, the sheer number of famous stories illustrates the importance engineering designers have placed on analogy.

In this article, we will examine three general questions about how analogies support a creative domain such as engineering design. (1) What kinds of analogies (close or distant) tend to get evoked during design? (2) How is the process of retrieving analogues influenced by the various forms of concrete-to-abstract objects found in design? and (3) What general functions do analogies serve in design? The answers to these questions will extend our understanding of analogical reasoning. In the sections that follow, we will expand on what is currently known and expected regarding these three questions.

\section{Analogical Distance}

In analogical transfer, the distance between the source and the target may be large or small. For example, a designer trying to develop door handles for the auto industry may make an analogy to other door handles in the auto industry (within-domain, or local, analogies) or may make an analogy to telephones or oysters in developing the design (between-domain, or distant, analogies; see Dunbar, 1995; Dunbar \& Blanchette, 2001; Vosniadou \& Ortony, 1989). Local analogies involve greater superficial similarity between the source and the target, as compared with the lesser amounts of superficial similarity involved in distant analogies. This increase in superficial similarity may make local analogies easier to access (e.g., Gentner, Rattermann, \& Forbus, 1993; Holyoak \& Koh, 1987). Both local and distant analogies involve structural similarity. But since distant analogies involve two vastly dif- 
ferent bodies of knowledge, it may be more difficult to ensure successful transfer of solution elements in design problem solving from the source to the target, because the domains may differ in multiple subtle ways (JohnsonLaird, 1989).

The use of distant analogies may be positively related to originality in design. Although experimental evidence on the subject is scarce, Dahl and Moreau (2002) found that the greater the percentage of distant analogies used during design, the more significantly positive the effect on the estimated originality of the design of the resulting product. Furthermore, in an experimental study of visual analogy in design, Casakin (2003) found that both novices and experts produced more between-domain than within-domain analogies. The experimental setup involved providing subjects with visually analogous displays and instructing them to use analogies, and this choice of experimental setup may have significantly affected the results. These research findings and anecdotal evidence would lead one to assume that distant analogies are very common in design and play an important part in the generation of creative products.

In science, similar anecdotal evidence of distant analogizing's leading to breakthrough discoveries exists (see, e.g., Ghiselin, 1954; Shepard, 1978). However, in his studies of real-world analogy in microbiology, Dunbar $(1995,2001 \mathrm{a})$ found that distant analogies did not play a significant part in discovery. Dunbar divided analogies into local, regional, and distant and found that distant analogies were very rare, in comparison with local and regional analogies.

So, it is unknown whether real-world designers use mainly within-domain or between-domain analogies. Insofar as the real-world science findings can be generalized, we would expect distant analogies to be rare in real-world design. But insofar as Casakin's experimental findings can be generalized, we would expect designers to use mainly distant analogies.

\section{Preinventive Structures and Analogy}

Preinventive structures are precursors for the final externalized creative products. Although the term preinventive structure typically refers to unsupported cognitive structures (e.g., visual patterns, object forms, or mental models; Finke, Ward, \& Smith, 1992), it may also refer to cognitive structures with the external support of sketches or prototypes. Creative cognitive processes in concept design are frequently supported by external representational systems, such as sketching (McGown, Green, \& Rodgers, 1998) and the construction of mock-ups and prototypes (Römer, Pache, Weisshahn, Lindemann, \& Hacker, 2001). In a survey of engineering designers, Römer et al. found that $96 \%$ reported using sketches and models during the development of solution concepts. Notably, this was also the case during the early stages of design (task clarification and concept design), where 95\% reported using sketches and 58\% reported using prototypes.

In some cases, preinventive structures may influence analogy use. It is well known that employing exemplars or fixating elements in either memory or the environment may constrain performance or creativity. Prime examples include such phenomena as functional fixedness, which involves restricting the uses of objects to well-known functions (Maier, 1931), and mental set, which involves situationally induced obstacles to problem solving (Luchins, 1942). Furthermore, it has been found that providing (Dahl \& Moreau, 2002; Jaarsveld \& van Leeuwen, 2005; Jansson \& Smith, 1991; Marsh, Bink, \& Hicks, 1999; Marsh, Ward, \& Landau, 1999; Ward, 1994) or retrieving (Ward, 1994) existing examples may inhibit generative creative processes and may lead to a higher proportion of property transfers from the examples into the subject's own work (e.g., Marsh, Landau, \& Hicks, 1996), even when the subject is explicitly instructed to avoid such transfer (e.g., Smith, Ward, \& Schumacher, 1993). Source monitoring of this property transfer is especially poor in generative tasks (e.g., Marsh, Landau, \& Hicks, 1997), which originally led to the label unconscious plagiarism, or cryptomnesia (Brown \& Murphy, 1989; Marsh \& Bower, 1993; Marsh \& Landau, 1995; Marsh et al., 1999). Such studies are usually not discussed under the analogical-reasoning heading, even though they do concern transfer of elements from previous examples into new creations.

Ward (1994, 1995, 1998) proposed a path-of-leastresistance model to account for some of these findings. This model states that the default approach in tasks involving the imagination, especially when few constraints must be satisfied, is to access a specific known entity or category exemplar and then to pattern the new entity after it. In support of this model, Ward (1994; Ward, Patterson, Sifonis, Dodds, \& Saunders, 2002) found that people who reported basing their novel constructions on specific exemplars were less original than people who used other strategies. Property transfer in generative tasks has proven robust across a variety of settings, including engineering design tasks conducted in the lab (Christiaans \& Andel, 1993; Dahl \& Moreau, 2002; Jansson \& Smith, 1991). Jansson and Smith had mechanical engineering students and professional designers work on simple design problems, such as how to construct a car-mounted bicycle rack, with (the fixation group) or without (the nofixation group) a specific example being provided by the experimenter. They found that the fixation group included more properties from the examples. (However, it should be noted that a failure to replicate this finding has been reported by Purcell \& Gero, 1992.) Following up earlier work, Dahl and Moreau had undergraduate engineering students design new products that would solve problems for the commuting diner (e.g., difficulties with spillage, consumption and storage of food during automotive driving). Subjects who saw an example sketch of a drive-in window tray transferred more properties of the example and generated fewer distant analogies than did the subjects who saw no sketch.

Taken together, the research on fixation and exemplar influence in generative tasks supports the notion that having or making examples available will bias people's creations toward features in those examples. In making 
these findings relevant to the analogy literature, it could be argued that since objects from similar domains share more superficial similarity than do objects from dissimilar domains and superficial similarity is one of the key driving forces of analogical access, we would expect that the presence or availability of within-domain exemplars would increase the likelihood of within-domain analogizing (Ward, 1998). In other words, the presence of within domain examples may make it hard for subjects to break away from local analogies, since superficial similarity dominates access and distant analogies will be less superficially similar than local analogies. Providing prior within-domain examples should thus bias people's creations toward features contained in those examples (Marsh et al., 1996). This within-domain biasing could, for example, be the case when designers use the external support of prototypes during the concept phases in engineering design, as compared with conditions without such support. However, it has never been tested whether exemplars that are a natural part of the design process, such as external support systems in the form of sketches or prototypes generated by the designers themselves, also constrain analogical distance. Some tentative support has come from experiments in which visual analogues were provided as hints in problem solving (Beveridge \& Parkins, 1987) and design (Bonnardel \& Marmèche, 2004; Casakin \& Goldschmidt, 1999), indicating that providing visual information can lead to the transfer of solution elements. Furthermore, Craig, Nersessian, and Catrambone (2002) examined the functioning of diagrams in analogical problem solving and found that aspects of drawings, such as view and configuration, can afford analogical transfer, but they did not focus on analogical distance.

These findings from the literature on fixation, exemplar influence in generative tasks, and analogical transfer lead to the following hypothesis: Use of within-domain external support, such as prototypes and sketching during the concept design phase, will result in a lower proportion of between-domain analogies, in comparison with using no such external support.

This prediction leaves open the question of whether there may be a difference in average analogical distance between external support relying on sketching and that relying on prototypes. If one compares sketches with prototypes, one could argue that sketching is characterized by density and ambiguity (Goel, 1995) and concentrates (like diagrams, but unlike drafting) primarily on illustrating the structural aspects of objects-to-be. As such, it could be argued that sketching involves less superficial similarity, as compared with prototypes. This argument leads to the hypothesis that sketches will result in higher proportions of between-domain analogies, relative to within-domain analogies, when compared with external supports with high resolution and superficial detail, such as prototypes.

\section{Functions of Analogy in Engineering Design}

Analogies are constructed for different purposes. In his studies of microbiology labs, Dunbar (1997, 2001a) distinguished four types of functions for analogies: forming hypotheses, designing experiments, fixing experiments, and explaining concepts to other scientists. However, these functions are, at least in part, specific to science and do not apply to design. Although engineering design certainly can involve experimentation, other kinds of activity are more prevalent and important, such as the construction, modification, and evaluation of novel and useful objects. Another classification of the function of analogies comes from Ward (1998), who classified analogies in invention or design as either explanatory or inventive. Along similar lines, Bearman, Ball, and Ormerod (2002) examined analogy, using a simulated real-world management decision-making task in a study involving undergraduate students. In this context, they distinguished between two different functions of analogies: problem solving and illustration. Both of these functions are well suited for the ill-structured domain of engineering design.

Adapting these prior findings to the design setting, we will distinguish three functions of analogies: explanation, problem solving, and problem identification. Engineering design is frequently conducted in teams, rather than individually, whereby communicating novel ideas to other members of a team becomes an important part of the process. Explanations through analogy can be a way of enhancing and ensuring comprehension, while avoiding misunderstanding when dealing with novelty. Thus, explanation or illustration using analogy is certainly a function to be expected in engineering design. Another function analogy is expected to serve is that of problem solving. Indeed, this function is perhaps the primary reason researchers have focused on analogy in design and science. In addition to these two functions, problem identification may be an important function, especially in the early conceptual stages of engineering design. When novel concepts are developed, it is necessary to try to foresee whether a novel idea or concept would work under particular circumstances. In this case, analogy may play some part in evaluating novel concepts, in that it is possible to transfer not only solutions, but also potential problems from sources with which the subject has had past experience. Here, the elements to be transferred from the source to the target involve potential design problems that the new concept may display.

The analogical functions may interact with external support. Communicative alignment (i.e., making sure everyone is talking about the same thing) quickly becomes a problem when novel ideas have to be shared among group members, because team members may not be certain that they are referring to the same not-yet-existing design object. It could be hypothesized that the need for communicative alignment will be greater when the design object takes the form of an idea than when external support is present, thereby producing more explanatory analogies.

The frequency of these three kinds of analogical functions in real-world design remains to be seen. On the basis of the results in Dunbar (1997, 2001a) and Bearman et al. (2002), we should at least predict that a substantial proportion of the analogies will involve explanations. In science, Dunbar found that almost half of the analogies 
were explanatory, and in management decision making, Bearman et al. found that $27 \%$ of the analogies served the function of illustration.

We predicted that analogical distance will interact in particular ways with analogical function. In science, Dunbar $(1997,2001 \mathrm{a})$ has argued that between-domain analogies are primarily explanatory in function. Betweendomain analogizing may be necessary in explaining novel design concepts exactly because the concept is new to the domain. Furthermore, problem identification in evaluation may involve primarily within-domain analogies. Within-domain analogies may be (1) more accessible, due to superficial similarity, (2) more available, due to within-domain expertise, and (3) more appropriate for identifying problems, because within-domain analogies may increase the chances of successful transfer. Finally, because engineering design involves the production of novel and useful solutions, solving problems by relating them to past within-domain knowledge may frequently not be enough to construct an original product. Therefore, a mixture of within- and between-domain analogies are to be expected when the function of the analogy is to solve a design problem.

\section{METHOD}

Data were collected using Dunbar's (1995, 1997; Dunbar \& Blanchette, 2001) in vivo methodology. In vivo methodology allows the researcher to study expert thinking and reasoning "online" in the real world. To this end, a major international company focusing on engineering design in the domain of medical plastics was selected for its persistent creativity displayed over many years. The company's research and development (R\&D) department had won multiple awards for a number of different designs. Upon contacting the R\&D department, a particular design project about to start up was chosen as the focus. The design project involved a total of 19 expert engineering designers organized into three subgroups focusing on different aspects of the design object and involved designing a new and improved product in a domain in which the company already had multiple products and extensive experience. The three subgroups were organized as multidisciplinary teams, involving different functions. The design project would span over 2 years.

When in vivo research is conducted, it is necessary to locate a suitable object of study (or time point) for the study of, in this case, design thinking and reasoning. A suitable object of study should include a broad cross-section of design activities, should include mainly design activities in the here and now (e.g., rather than retrospective accounts of designing), and should involve natural dialogue between the designers so as to avoid potentially problematic think-aloud instructions (see also Christensen, 2005). For example, Kevin Dunbar studied scientific thinking and reasoning by recording lab group meetings because he found that they "provided a far more veridical and complete record of the evolution of ideas than other sources of information" (Dunbar, 2001b, p. 120).
An analogous object of study in design turned out to be subgroup product development meetings. Each subgroup in the project held product development meetings on a regular basis (e.g., weekly). Because the designers were talking out loud, there was an external record of thinking and reasoning. The primary function of these subgroup product development meetings was the creative development of design artifacts - that is, actual creating and problem solving in collaboration-and the activity included brainstorming, developing concepts, solving design problems, planning data collection and the next steps of the design process, testing and evaluating mock-ups and prototypes, sketching activity, conducting experiments, and discussing and exchanging knowledge about end users and production methods. Furthermore, it was found that the vast majority of the design activity at these meetings concerned design thinking and reasoning in the here and now. Therefore, the subgroup product development meetings were chosen as the most suitable object of study.

In the present article, the meetings of one particular subgroup will be examined. This subgroup had the task of developing completely novel features for the new product and was chosen over the other subgroups working on improving existing features, due to the expectation that the new feature task requirement would maximize the density of creative cognitive elements. It consisted of 5 core members ( 1 of them female and 4 male) representing different functions (industrial designer, lab technician, project manager) and backgrounds (machine engineering, architecture, machinist). They all had extensive experience in medical plastics and design (10, 10, 20, 27, and 35 years). Besides these core members, the team would invite experts with specialized knowledge from other parts of the company to participate in the group meetings, when required.

The subgroup product development meetings were videotaped using a single camera capturing design objects present on the table between the designers and object handling (e.g., holding prototypes or sketching activity), albeit not in detail. Furthermore, gestures and the general directions of gaze of the designers could be discerned from the video. During the meetings, the experimenter was present as an observer only. No special instruction to think aloud was given. The designers were merely asked to continue with the meeting as they normally would. Following each meeting, design objects (sketches and prototypes) that had been present were videotaped in close-up, sometimes with one of the designers explaining the functioning of the object in voice-over.

Each meeting lasted between $30 \mathrm{~min}$ and $2 \mathrm{~h}$. Subgroup product development meetings were recorded during the concept phase, or first 5 months, of the design project. This time frame was chosen in order to maximize the density of the creative cognition data. The recordings were transcribed and segmented according to complete thought. A total of seven transcripts, covering approximately $9 \mathrm{~h}$ of video, were used in the present data analysis, yielding a total of 7,414 segments.

Each segment was supplemented with information regarding which (if any) design object presently in the room 
was the focus of attention of the person speaking (typically, a design object, such as a sketch or prototype located on the table between the designers). This information was coded for each segment, using the video recording of the design session (not the verbal data). Focus of attention was operationalized as either actual handling or holding a particular object, pointing to a particular object, or gazing toward a particular object (if this was possible to discern from the video). The main unit of analysis was the segmented data.

The designers developed multiple design concepts, and most were discarded again. The designers would work on several different design concepts at each meeting, although usually two or three would be the main focus of each session.

\section{Protocol Coding}

The transcripts were initially reduced by coding for offtask behavior (e.g., jokes, banter between the designers, office gossip, or events unrelated to design) and episodes dealing with summarizing past meetings or planning future meetings or data collection. This coding removed 1,602 segments from further analysis. Then the transcripts were coded for type of preinventive structure and analogy. All analogies were then coded further for analogical distance and analogical purpose. All the transcripts were coded by the first author. Reliability checks were conducted by an independent coder who had received training both in protocol analysis in general and in this coding scheme, using spare data from a different subgroup.

\section{Coding of Type of Preinventive Structure}

For each segment, we coded whether the verbally referenced design object was unsupported by external representations (idea), supported by sketches (sketch), or supported by 3-D physical objects in the form of prototypes (prototype). These types of design objects in the making will be referred to as preinventive structures (Finke et al., 1992). These three types exhausted the types of design objects found in the transcripts. In addition, a finalized existing product (other) category was used to capture references solely to existing products on the market, either from the same company or from a competitor. These other segments, referring exclusively to products already on the market, were excluded from further analysis because they were not considered creative design objects in the making. Note that this coding scheme did not exclude analogy segments in which existing products were used as a source to create a novel concept, because in that case, the novel concept was considered a preinventive structure.

\section{Coding of Analogy}

All the segments were coded for analogies, following the method developed by Dunbar $(1995,1997)$. Any time a designer referred to another base of knowledge to explain, create, modify, or evaluate a design, it was coded as an analogy. An analogy was defined as consisting of both an explicit mapping and an explicit transfer. Mapping and transfer can take place in separate segments. For example, an analogy is a statement such as "This reminds me of one of John's old ideas, where he put holes in the side of the box. Could you do something similar here?" Here, the first sentence maps the old idea to the present context, and the second sentence ensures explicit transfer from the old idea to the new one. Without the last sentence (in italics), this example cannot be counted as an analogy, due to a lack of explicit transfer. Without explicit structural transfer, the mapping may lead to nothing more than a statement of similarity (A is like B).

\section{Coding of Analogical Distance}

All the analogies were coded for analogical distance. Two levels of analogical distance were used: within domain and between domain. Within-domain analogies were defined as analogical mappings within the domain of medical plastics (for example, to existing products from the same company or from competitors). Betweendomain analogies were analogies made to domains outside of medical plastics (for example, to the auto industry, biology, or sports).

\section{Coding of Analogical Function}

All the analogies were coded for one of three functions. The function could concern identifying possible problems in a new design, taken from an analogous source (identify problem). Another function could involve solving design problems (solve problem), where the transfer from source to target included elements that would potentially solve a particular design problem that the target was having. Finally, the function of the analogy could be to explain a design to the other designers (explain). All the analogies could be classified using these three categories.

\section{RESULTS}

\section{Interrater Reliability}

Reliability coding was done on two full transcripts (approximately $18 \%$ of the data). All disagreements between coders were resolved through discussion. For each code, a reliability kappa coefficient was calculated: type of preinventive structure, .73; analogy, .71; analogical distance, 1.00; analogical function, .80. All the codes reached a satisfactory level $(>.70)$, with perfect agreement for analogical distance.

\section{Frequency of Analogies}

A total of 102 analogy segments were found in the seven transcripts $(M=11.3$ analogy segments per hour of verbal data). The range of analogy segments was 4-21 per transcript, showing that analogies were commonly used by the designers during product development meetings.

\section{Analogical Distance}

The analogies were $55 \%$ within and $45 \%$ between domain. All the transcripts contained both within-domain analogies and between-domain analogies. It thus appears that within-domain and between-domain analogies were used in roughly similar quantities and that both occurred 
frequently. Examples of between-domain sources included potato print, zippers, credit cards, children's slides, milk containers, shoes, toilet paper, cars, Christmas decorations, water wheels, picture puzzles, Venetian blinds, and lingerie. In short, a large number of distant domains, seemingly with little or no relation or superficial similarity to medical plastics, were accessed and used during design problem solving.

To substantiate the theoretical claim that within-domain analogies shared more superficial similarity than did between-domain analogies in the present sample, we computed whether each analogical source and target shared four different superficial similarities: basic size, basic shape, basic color, and basic materials used. No between-domain analogy shared all of these four features, whereas $85 \%$ of the within-domain analogies shared all four features. On the other hand, $61 \%$ of the between-domain analogies shared none of the four superficial similarity features, as compared with zero cases for within-domain analogies. An independent $t$ test revealed that within-domain analogies $(M=3.82)$ had significantly more shared superficial features than did between-domain analogies $(M=0.52)$ $[t(100)=17.21, p<.001]$. Although these four features are not a complete list of superficial similarities, this very large difference in four basic features does lend support to the claim that there is a close relationship between degree of superficial similarity and domain specificity in analogy in the present sample.

\section{Preinventive Structures and Analogy Use}

Figure 1 presents the percentages of analogies in segments associated with ideas, sketches, and prototypes. Chi-square results indicated that there were significant differences between conditions [ $\left.\chi^{2}(2)=7.96, p<.019\right]$. A number of subsequent $2 \times 2$ chi-square analyses were

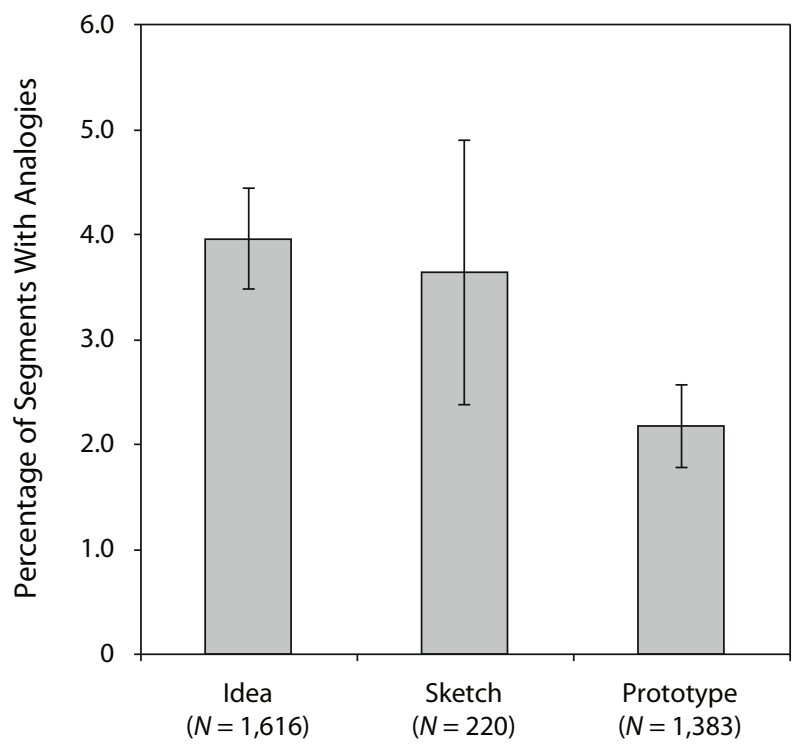

Type of Preinventive Structure

Figure 1. Percentages of segments with analogies by type of preinventive structure (with standard error bars). conducted on each individual pair of preinventive structures (idea vs. sketch, idea vs. prototype, and sketch vs. prototype) $\times$ presence versus absence of analogies. These analyses indicated that ideas had a significantly higher percentage of analogies than did prototypes $\left[\chi^{2}(1)=7.88\right.$, $p<.006]$, whereas sketches did not differ significantly from either ideas or prototypes (see Figure 1). The designers thus produced more analogies when referring to unsupported ideas than when they referred to prototypes.

To examine the relation between analogical distance and preinventive structure, a chi-square between analogical distance (between domain, within domain, or no analogy) and preinventive structure (ideas or prototypes) was conducted. Sketches were removed from this analysis, due to their having an expected count of less than five. The results showed significant differences in analogical distance $\left[\chi^{2}(2)=19.26, p<.001\right]$. The same number of within-domain analogies were produced for idea and prototype segments, but significantly more between-domain analogies were produced for idea segments than for prototype segments (see Figure 2). The overall result, that ideas were linked to more analogies in total than were prototypes, is therefore attributable to prototypes' having fewer between-domain analogies.

To examine whether the ratio of within- to betweendomain analogies differed between sketches, ideas, and prototypes, Fisher's exact tests were conducted on the number of within- and between-domain analogies for each pair of preinventive structures (see Figure 3), thereby eliminating the no-analogy baseline segments. The results indicated that, as before, ideas differed from prototypes (two-tailed Fisher's exact test, $p<.001$ ), with more betweendomain analogies for ideas. Furthermore, sketches differed from prototypes (two-tailed Fisher's exact test, $p<.019$ ), with sketches producing more between-domain analogies, relative to within-domain analogies. Sketches did not differ significantly from ideas (two-tailed Fisher's exact test, $p=1.0$ ). Thus, both ideas and sketches were associated with more between-domain analogies, relative to withindomain analogies, as compared with prototypes.

\section{Analogical Function}

The functions of the analogies were distributed roughly evenly across the three categories, with $28 \%$ of the analogy segments focusing on identifying problems, $40 \%$ on solving problems, and the remaining $32 \%$ on explaining. Analogy thus regularly served each of the three functions in the present data set.

To examine whether the ratio of within- and betweendomain analogies varied with analogy function, a chi-square test was conducted (see Figure 4), revealing significant differences between the three types of functions $\left[\chi^{2}(2)=\right.$ $20.93, p<.001]$. Subsequent $2 \times 2$ chi-squares (each individual pair of functions $\times$ proportion of between-domain analogies) revealed that there were significant differences between all the pairs of analogical functions: identify problem versus solve problem $\left[\chi^{2}(1)=6.72, p<.01\right]$, solve problem versus explain $\left[\chi^{2}(1)=6.19, p<.02\right]$, and identify problem versus explain $\left[\chi^{2}(1)=20.83, p<.001\right]$. As was predicted, the results indicate that analogies used to identify 


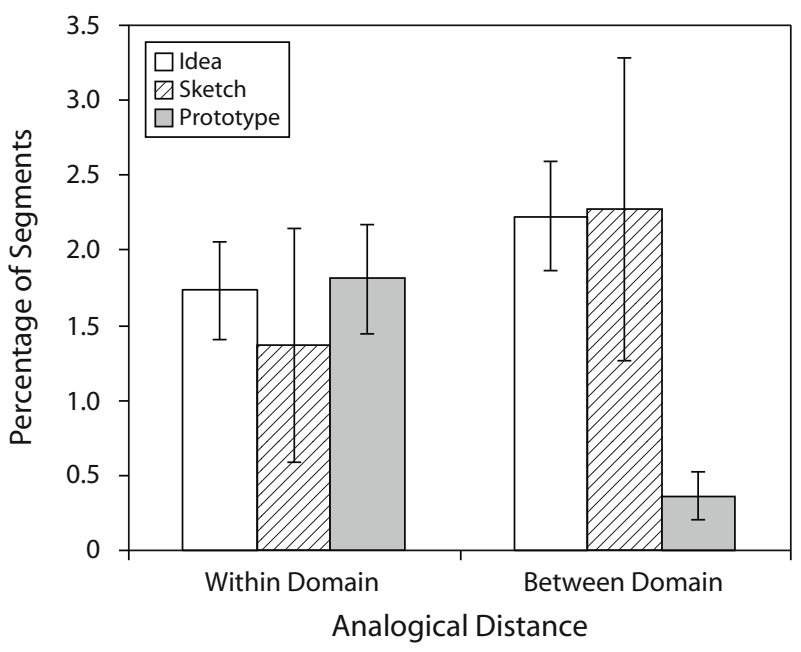

Figure 2. Percentages of segments containing within-domain and between-domain analogies in the data set by preinventive structure (with standard error bars).

problems primarily utilized within-domain sources, whereas explanatory analogies mainly used between-domain sources and problem-solving analogies used a mixture of withinand between-domain analogies.

A category of special interest to design problem solving is the category related to the solving of problems by utilizing between-domain analogies. This category has traditionally been linked to radical novelty in design problem solving, as evidenced in anecdotes. Two examples will be provided to illustrate this kind of analogy in the present data set. ${ }^{1}$

The designers were trying to create a kind of portable slide or tube that could be used to transport liquid from one container to another at a somewhat shallow slope without support. At the same time, the device had to be soluble in water over a few hours or days. However, these requirements raised problems, in that at least two of the constraints appeared to be in conflict. Portability required the device to be either somewhat small or at least foldable. But the function of supporting liquid at a shallow angle meant that the obvious choice of material was a hard and sturdy one. Similarly, there was a conflict between immediate durability and solubility: how to combine hard and sturdy with foldable and soluble. This conflict led to the following exchange:

\section{Designer}

A what if you made ... now I'm just saying something ... the stuff you make Venetian blinds of for example ... they can be bent.

B yes they can...

A ... folded ...

B Yes

A ... and when you ... when they want to ... but you can't dump them in water
B No, 'cause they're made of steel

A But they are ... they have this curve, they can be folded into next to nothing ... it's

B Yes, that's right, but it is . . it is really really thin steel, and that's why it goes click-click ...

Following this exchange, the designers left the Venetian blinds analogy, not to return. The problem solving attempt apparently failed, even though several constraints were met by the analogy (foldable; solid and sturdy), because a further constraint (water soluble) was not met by the suggested analogy.

Another example of between-domain problem-solving analogies involved a different design concept. Again, a design requirement was that it had to be water soluble, but this time, the design involved a container for small amounts of liquid, capable of holding the liquid for a few minutes before falling apart. The problem was finding a suitable material for the design. Most plastics the team discussed were too durable. The following exchange ensued:

Designer

A But ... what's it called ... one of these things ... [points to an envelope] . . . if you made it in a paper bag, that will take some time ... maybe ... perhaps you should ... if you could make it look flushable...

B ... but it's going to be hard to flush

A Well yes but you could ... it has to be made flushable...

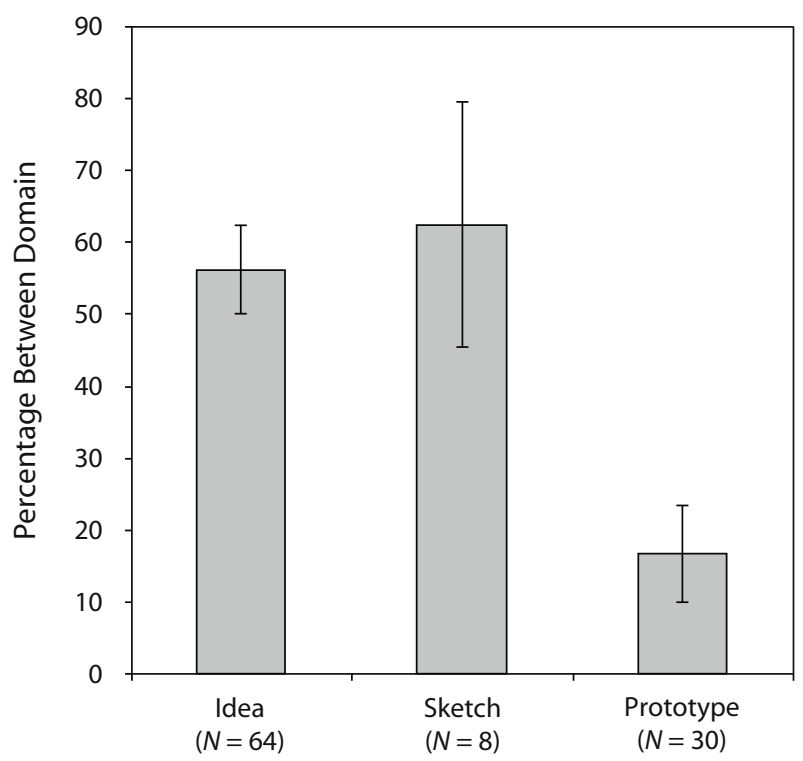

Type of Preinventive Structure

Figure 3. Percentages of analogies that were between-domain by preinventive structure (with standard error bars). 


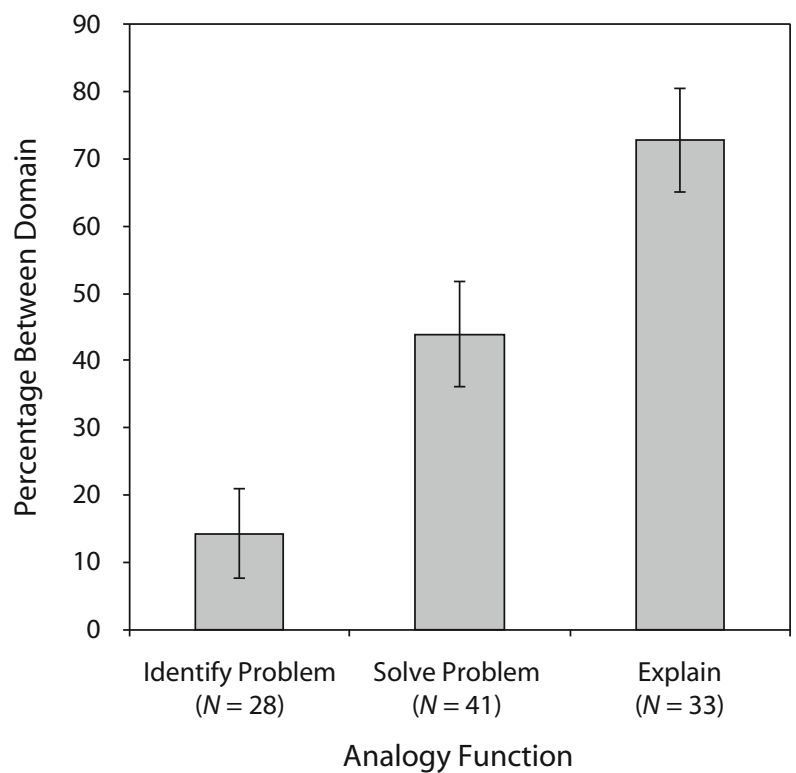

Figure 4. Percentages of between-domain analogies by analogical function (with standard error bars).

\section{We're going to make it out of paper!}

The designers worked on this idea for a novel kind of material for their product for quite some time to follow, conducting experiments by using the envelope mentioned in the transcript. Later in the meeting, it was decided to examine different types of paper and to develop prototypes of the product. Apparently, the analogy to the envelope was a successful one. In conclusion, the designers did use between-domain analogies in their active problem solving, supporting the view that distant analogies may play an important part in engineering design. However, it should be noted that analogies also served functions other than solving problems (such as explaining concepts and identifying problems). Furthermore, within-domain analogies were used just as frequently in solving problems, showing that between-domain analogies were not the only type of analogies used in solving problems.

\section{Communicative Alignment}

To examine whether or not more explanatory analogies would be found when unsupported ideas were used, due to the absence of any external referents, explanatory analogies for ideas versus prototypes were compared, using a chi-square test, excluding problem identification and problem-solving analogies. Chi-square tests could not be conducted for the sketch category, due to low- $N$ problems (7 out of 219 segments were explanatory analogies), so the sketch category was excluded from the analysis. Comparing ideas with prototypes did yield a significant result in the expected direction $\left[\chi^{2}(1)=13.01, p<.002\right.$; see Figure 5].

The significant relation between explain analogies and idea versus prototype segments could have interacted with the previous result on the relation between analogical distance and preinventive structures, since explain analogies are mainly between domain. To examine whether the higher proportion of explain analogies for ideas than for prototypes interacted with, and perhaps created, the relationship between analogical distance and preinventive structure, we excluded all explain analogies from the data set and again ran an analysis on the relation between analogical distance and preinventive structure. The results were significant in the expected direction $\left[\chi^{2}(1)=8.33, p<.004\right]$, with ideas still producing more between-domain analogies than did prototypes, indicating that the interaction with analogical function did not create the relation between analogical distance and preinventive structure.

\section{Temporal Development or Order Confound Tests}

In comparing sketches and prototypes in a naturalistic setting, it is important to consider whether there is an inherent temporal or order confound. That is, can any difference in performance with ideas versus sketches versus prototypes be attributed to when they are used in the design process? For example, unsupported ideas may be more prevalent in the early stages of problem solving, whereas prototypes may be more prevalent at the later stages, with sketching somewhere in between. This might lead to confounds in the present results and to spurious associations among the variables. We examined this potential confound empirically. All significant results were reanalyzed by temporal halves. The data were split in two ways. First, to test for temporal effects between transcripts, the transcripts were ranked according to data collection date, and the first half of the collected transcripts was separated from the second half. Second, to test for temporal effects within each transcript, each transcript was split into two halves. Under both approaches, the prior analyses were conducted for the first halves and the second halves separately. In the majority of cases, each half of the data yielded significant results in the same direction as that for the total data set. In some cases, due to low- $N$ problems, one of the halves did not yield significant results or chi-square tests could not be conducted, due to expected counts of less than five. But it is important to note that in all cases, the directionality of

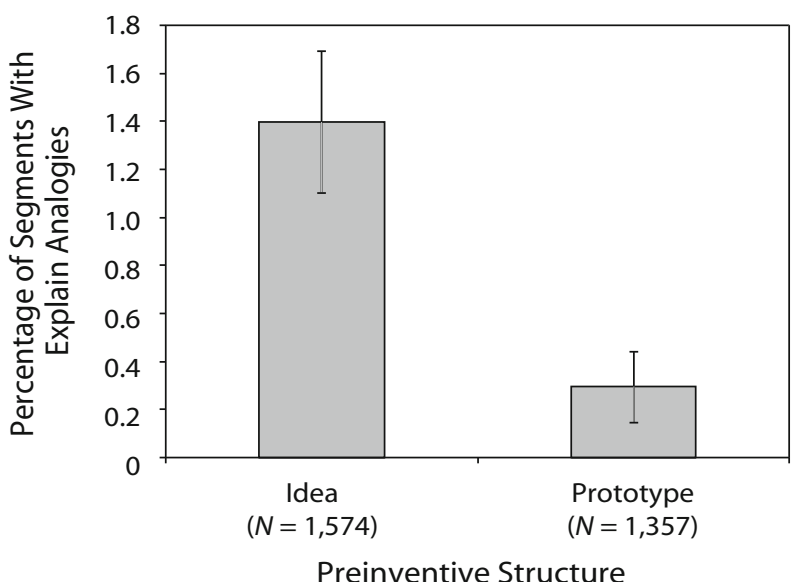

Figure 5. Percentages of segments with explain analogies by preinventive structure (with standard error bars). 
the data was the same as that for the total data set. Thus, time course confounds could not explain our results.

\section{DISCUSSION}

In the present study, we examined the design processes of a creative, real-world design team in depth to test the interaction between preinventive structures and analogical transfer of different forms. Although the overall base rates of different activities may be peculiar to this particular team or to the particular objects that they were designing, we take the patterns of the functional roles of preinventive structures with respect to analogical transfer to be a general, albeit preliminary, picture of design cognition.

The present study showed that, unlike in real-world science, between-domain analogies are quite frequent in engineering design and almost as frequent as within-domain analogies, suggesting that they serve important functions in design cognition (especially for explanations, but also for problem solving), as would be predicted from the large number of anecdotes and design tools claiming that between-domain analogies are crucial to design. But as has been found in the domain of science, within-domain analogies also play an important role in design.

More analogies were made when the designers were not using external representations than when they were referring to prototypes. This finding could be explained by a differential number of between-domain analogies between ideas and prototypes. Previous studies and theories have suggested that making within-domain exemplars available during the creative constructive process tends to lead subjects to unconsciously plagiarize these exemplars when they try to construct novel objects. The present research extended this finding by showing that the within-domain exemplars may even be the designers' own prototypes constraining the creative process, as evidenced in a reduction in the number of between-domain analogies the designers made. When the designers were referring to prototypes, they made very few between-domain analogies, and notably fewer than when referring to either sketches or ideas unsupported by external representations. This finding supports the hypothesis that within-domain exemplars constrain creativity by providing paths of least resistance for design analogizing. In other words, if exemplars are present, the designers are less likely to think about domains other than the present one. Apparently, constraining exemplars in creativity include not only accidental exemplars in the immediate environment, but also external representations made and used by the designers themselves to (paradoxically) help the creative process along! Notably, however, sketching appeared not to constrain analogical distance in the present data set, which could, perhaps, explain in part why designers are so prone to sketching.

This finding has potentially important implications for how the early stages of design should be structured. In so far as the designer's current design goal involves generating novel and original products, a tentative recommendation could be to use sketching and idea generation unsupported by external representation in the early stages of design and, perhaps, to postpone prototyping until several promising concepts have been developed.

The functions of analogy in engineering design, as revealed by the present study, include explanation, problem solving, and problem identification. As has been found in previous research in science, a significant part of the analogies made had an explanatory function in relation to explaining concepts to other designers. It is possible that one of the primary reasons for the importance of explanatory analogies is that in design involving the development of novel concepts, communicative alignment in a group becomes a main concern when the object being referenced exists only in the mind of other team members. Indeed, ideas did have more explanatory analogies than did prototypes. A large proportion of analogies were made to solve design problems, as would be expected on the basis of previous research and the conclusions of design theorists. But support for a third and new function was also found, in that several analogies concerned identifying problems in novel designs. Here, rather than transferring solutions to the novel design, what was transferred was the expected existence of potential design problems, taken from past sources with which the designers had experience. This type of analogical transfer served a function in the quick evaluation of newly developed design concepts.

These three types of analogy functions in design had differential ratios of within- to between-domain analogies. As was hypothesized, problem identification analogies were mainly within domain, explanatory analogies were more frequently (and mainly) between domain, and problemsolving involved a mixture of within- and between-domain analogies. Unlike Dunbar's (1995, 2001a) findings in science, between-domain analogies not only were made to explain concepts, but concerned problem solving as well. These results indicate the importance of between-domain analogies in real-world engineering design, as has also been suggested by anecdotes and design techniques, but at the same time, the results stress that analogy serves several other functions in design.

Overall, this work has expanded our understanding of analogical reasoning, showing how it interacts with the function of the analogy and the external environment of the problem solver.

\section{AUTHOR NOTE}

Correspondence concerning this article should be addressed to B. T. Christensen, Department of Marketing, Copenhagen Business School, Solbjerg Plads 3, 2000 Frederiksberg, Denmark (e-mail: bo@ virksom .dk).

\section{REFERENCES}

Bearman, C. R., Ball, L. J., \& Ormerod, T. C. (2002). An exploration of real-world analogical problem solving in novices. In W. D. Gray \& C. D. Schunn (Eds.), Proceedings of the 24th Annual Conference of the Cognitive Science Society (pp. 101-106). Mahwah, NJ: Erlbaum. Beveridge, M., \& Parkins, E. (1987). Visual representation in analogical problem solving. Memory \& Cognition, 15, 230-237.

BONNARDEL, N., \& MARMĖCHE, E. (2004). Evocation processes by novice and expert designers: Towards stimulating analogical thinking. Creativity \& Innovation Management, 13, 176-186.

BROWN, A. S., \& MURPHY, D. R. (1989). Cryptomnesia: Delineating 
inadvertent plagiarism. Journal of Experimental Psychology: Learning, Memory, \& Cognition, 15, 432-442.

CASAKIN, H. (2003). Visual analogy as a cognitive strategy in the design process: Expert versus novice performance. In N. Cross \& E. Edmonds (Eds.), Expertise in design. Sydney: University of Technology, Creativity \& Cognition Press.

Casakin, H., \& Goldschmidt, G. (1999). Expertise and the use of visual analogy: Implications for design education. Design Studies, 20, 153-175.

CHRISTENSEN, B. T. (2005). A methodology for studying design cognition in the real-world [Online]. In Digital proceedings from NORDES: The First Nordic Design Research Conference. Copenhagen.

ChristiaAns, H., \& Andel, J. v. (1993). The effects of examples on the use of knowledge in a student design activity: The case of the "flying Dutchman." Design Studies, 14, 58-74.

Craig, D. L., Nersessian, N. J., \& Catrambone, R. (2002). The role of diagrams and diagrammatic affordances in analogy. In W. D. Gray \& C. D. Schunn (Eds.), Proceedings from the 24th Annual Meeting of the Cognitive Science Society (pp. 250-255). Mahwah, NJ: Erlbaum.

DAhL, D. W., \& Moreau, P. (2002). The influence and value of analogical thinking during new product ideation. Journal of Marketing Research, 39, 47-60.

Dunbar, K. (1995). How scientists really reason: Scientific reasoning in real-world laboratories. In R. J. Sternberg \& J. E. Davidson (Eds.), The nature of insight (pp. 365-395). Cambridge, MA: MIT Press.

Dunbar, K. (1997). How scientists think: On-line creativity and conceptual change in science. In T. B. Ward, S. M. Smith, \& J. Vaid (Eds.), Creative thought: An investigation of conceptual structures and processes (pp. 461-493). Washington, DC: American Psychological Association.

Dunbar, K. (2001a). The analogical paradox: Why analogy is so easy in naturalistic settings yet so difficult in the psychological laboratory. In D. Gentner, K. J. Holyoak, \& B. N. Kokinov (Eds.), The analogical mind: Perspectives from cognitive science (pp. 313-334). Cambridge, MA: MIT Press.

Dunbar, K. (2001b). What scientific thinking reveals about the nature of cognition. In K. Crowley, C. D. Schunn, \& T. Okada (Eds.), Designing for science: Implications from everyday, classroom, and professional settings (pp. 115-140). Mahwah, NJ: Erlbaum.

Dunbar, K., \& Blanchette, I. (2001). The in vivo/in vitro approach to cognition: The case of analogy. Trends in Cognitive Sciences, $\mathbf{5}$, 334-339.

FinKE, R. A., WARD, T. B., \& SMith, S. M. (1992). Creative cognition. Cambridge, MA: MIT Press.

Gentner, D. (1998). Analogy. In W. Bechtel \& G. Graham (Eds.), A companion to cognitive science (pp. 107-113). Malden, MA: Blackwell.

Gentner, D., Rattermann, M. J., \& Forbus, K. D. (1993). The roles of similarity in transfer: Separating retrievability from inferential soundness. Cognitive Psychology, 25, 524-575.

GHISELIN, B. (1954). The creative process: A symposium. Berkeley: University of California Press.

Goel, V. (1995). Sketches of thought. Cambridge, MA: MIT Press, Bradford Books.

GoldschmidT, G. (2001). Visual analogy: A strategy for design reasoning and learning. In C. M. Eastman, W. M. McCracken, \& W. C. Newstetter (Eds.), Design knowing and learning: Cognition in design education (pp. 199-220). Amsterdan: Elsevier.

Gordon, W. J. J. (1961). Synectics: The development of creative capacity. New York: Harper \& Row.

HolyOAK, K. J., \& KoH, K. (1987). Surface and structural similarity in analogical transfer. Memory \& Cognition, 15, 332-340.

HolyOAK, K. J., \& THAGARD, P. (1995). Mental leaps: Analogy in creative thought. Cambridge, MA: MIT Press.

JaARSveld, S., \& VAN LEeuwen, C. (2005). Sketches from a design process: Creative cognition inferred from intermediate products. $\mathrm{Cog}$ nitive Science, 29, 79-101.

Jansson, D. G., \& Smith, S. M. (1991). Design fixation. Design Studies, 12, 3-11.

JoHNSON-LAIRD, P. N. (1989). Analogy and the exercise of creativity. In S. Vosniadou \& A. Ortony (Eds.), Similarity and analogical reasoning (pp. 313-331). Cambridge: Cambridge University Press.

LuCHINs, A. S. (1942). Mechanisation in problem solving: The effect of Einstellung. Psychological Monographs, 54(Whole No. 248).
MAIER, N. R. F. (1931). Reasoning in humans: II. The solution of a problem and its appearance in consciousness. Journal of Comparative Psychology, 8, 181-194.

Marsh, R. L., BinK, M. L., \& Hicks, J. L. (1999). Conceptual priming in a generative problem-solving task. Memory \& Cognition, 27, 355-363.

MARSH, R. L., \& BowER, G. H. (1993). Eliciting cruptomnesia: Unconscious plagiarism in a puzzle task. Journal of Experimental Psychology: Learning, Memory, \& Cognition, 19, 673-688.

MARSH, R. L., \& LANDAU, J. D. (1995). Item availability in cryptomnesia: Assessing its role in two paradigms of unconscious plagiarism. Journal of Experimental Psychology: Learning, Memory, \& Cognition, 21, 1568-1582.

MARSH, R. L., LANDAU, J. D., \& Hicks, J. L. (1996). How examples may (and may not) constrain creativity. Memory \& Cognition, 24, 669-680.

MARSH, R. L., LANDAU, J. D., \& Hicks, J. L. (1997). Contributions of inadequate source monitoring to unconscious plagiarism during idea generation. Journal of Experimental Psychology: Learning, Memory, \& Cognition, 23, 886-897.

MARSH, R. L., WaRD, T. B., \& LANDAU, J. D. (1999). The inadvertent use of prior knowledge in a generative cognitive task. Memory \& Cognition, 27, 94-105.

McGown, A., Green, G., \& Rodgers, P. A. (1998). Visible ideas: Information patterns of conceptual sketch activity. Design Studies, 19, 431-453.

PURCELL, A. T., \& GERo, J. S. (1992). Effects of examples on the results of a design activity. Knowledge-Based Systems, 5, 82-91.

Römer, A., Pache, M., Weisshahn, G., Lindemann, U., \& Hacker, W. (2001). Effort-saving product representations in design —results of a questionnaire survey. Design Studies, 22, 473-491.

Roozenburg, N. F. M., \& EeKels, J. (1996). Product design: Fundamentals and methods. Chichester, U.K.: Wiley.

SHEPARD, R. N. (1978). Externalization of mental images and the act of creation. In B. S. Randawa \& W. E. Cofman (Eds.), Visual learning, thinking, and communication (pp. 133-189). New York: Academic Press.

Smith, S. M., Ward, T. B., \& Schumacher, J. S. (1993). Constraining effects of examples in a creative generation task. Memory \& Cognition, 21, 837-845.

TERNINKO, J., ZuSMAN, A., \& Zlotin, B. (1998). Systematic innovation An introduction to TRIZ. Boca Raton: St Lucie Press.

Vosniadou, S., \& ORTONY, A. (1989). Similarity and analogical reasoning: A synthesis. In S. Vosniadou \& A. Ortony (Eds.), Similarity and analogical reasoning (pp. 1-7). Cambridge: Cambridge University Press.

WARD, T. B. (1994). Structured imagination: The role of category structure in exemplar generation. Cognitive Psychology, 27, 1-40.

WARD, T. B. (1995). What's old about new ideas? In S. M. Smith, T. B. Ward, \& R. A. Finke (Eds.), The creative cognition approach (pp. 157178). Cambridge, MA: MIT Press.

WARD, T. B. (1998). Analogical distance and purpose in creative thought: Mental leaps versus mental hops. In K. J. Holyoak, D. Gentner, \& B. N. Kokinov (Eds.), Advances in analogy research: Integration of theory and data from the cognitive, computational, and neural sciences (pp. 221-230). Sofia: New Bulgarian University.

Ward, T. B., Patterson, M. J., Sifonis, C. M., Dodds, R. A., \& SaunDERS, K. N. (2002). The role of graded category structure in imaginative thought. Memory \& Cognition, 30, 199-216.

\section{NOTE}

1. The examples do not provide exact details about the product in question, to ensure anonymity for the company, but care has been taken to correctly illustrate the structural requirements. Except to change two concepts that could have endangered anonymity, the protocol excerpts have not been altered in any way.

(Manuscript received April 1, 2005; revision accepted for publication November 3, 2005.) 